\title{
Identification of Exon 1 of Prostase/KLK-L1 Gene
}

\author{
CHRISTINA V. OBIEZU, ${ }^{1,2}$ GEORGE YOUSEF, ${ }^{1,2}$ and ELEFTHERIOS P. DIAMANDIS ${ }^{1,2}$ \\ ${ }^{1}$ Department of Pathology and Laboratory Medicine, Mount Sinai Hospital, Toronto, Ontario, Canada, \\ and ${ }^{2}$ Department of Laboratory Medicine and Pathobiology, University of Toronto, Toronto, Ontario, \\ Canada
}

\section{Introduction}

$\mathbf{P}$ rostatic neoplasia is the most commonly diagnosed cancer in North American men. While such markers as prostate specific antigen (PSA) and human glandular kallikrein 2 (hK2) have been available for some time to screen and diagnose this disease, their non-specificity for prostate cancer and their inability to differentiate between aggressive and clinically unimportant forms of this malignancy create the need for the identification of additional, more specific biomarkers. The identification of genes selectively expressed in the human prostate is a key step in furthering these efforts. Prostase/KLK-L1 is one such gene, discovered concurrently by our group using the positional candidate gene approach (1), and by Nelson et al. (2), using a subtractive hybridization approach. This novel serine protease is now known to colocalize with PSA and hK2 to chromosome 19q13.3-13.4 (1) and to be upregulated by androgens in the LNCaP cell line (2) and by both androgens and progestins in the breast carcinoma cell line BT-474 (3). The similarities between prostase/KLK-L1, PSA, and hK2 suggest that this gene may be a new candidate prostatic biomarker that may also play a role in the pathogenesis of prostatic malignancy. Genomic sequence analysis of this gene $(1,2,4)$ predicted the presence of five exons; however, its PCR amplification from a human prostatic cDNA library (2) and EST libraries (1) could only confirm the presence of exons $2-5$. The full characterization of this gene, and its encoded protein, requires knowledge of its first exon. Consequently, this study aimed to gain definitive evidence of the location and sequence identification of exon 1 of this gene.

Correspondence: E. P. Diamandis, M.D., Ph.D., F.R.C.P.C., Department of Pathology and Laboratory Medicine, Mount Sinai Hospital, 600 University Avenue, Toronto, Ontario, Canada M5G 1X5. E-mail: ediamandis@mtsinai. on.ca.

Manuscript received November 18, 1999; revised January 28, 2000; accepted February 2, 2000.

\section{Methods}

\section{Preparation of PRostatic tissue cDNA}

Using $2 \mu \mathrm{g}$ of total RNA isolated from human prostatic tissue (Clonetech Laboratories, Palo Alto, CA), first strand cDNA synthesis was carried out by using the SuperScript Preamplification System, as prescribed by the manufacturer (Gibco BRL, Life Technologies, Burlington, Canada). To test the success of cDNA synthesis, $1 \mu \mathrm{L}$ of the reverse transcription product was amplified using polymerase chain reaction (PCR) with primers designed to specifically amplify actin cDNA. Product was visualized on a $2 \%$ agarose gel stained with ethidium bromide.

PRIMER DESIGN AND AMPLIFICATION OF PROSTASE/KLKL1

In order to amplify the putative exon 1 , we assumed its sequence, predicted by informatics $(1,2)$, to be correct. Based on this assumption, the forward primer PRSTZ F1 (5' ATG GCC ACA GCA GGA AAT CC $3^{\prime}$ ) and reverse primer PRSTZ R1 (5' ACG GTA GGC ATT CTG CCG TT 3') were synthesized. These two primers were designed to amplify the prostase/KLK-L1 cDNA starting from the start codon in exon 1 up to the beginning of exon 4, producing a 491 base pair PCR product.

PCR reaction was prepared using $17 \mu \mathrm{L}$ deionized water, $2.5 \mu \mathrm{L}$ of $25 \mathrm{mM} \mathrm{MgCl}_{2}, 1 \mu \mathrm{L}$ of $5 \mathrm{mM}$ deoxyribonucleotide triphosphate mixture, $0.5 \mu \mathrm{L}$ $(\sim 150 \mathrm{ng})$ of each of the primers, $0.25 \mu \mathrm{L}$ of Taq Gold DNA polymerase (Roche, Laval, Canada), and $1 \mu \mathrm{L}$ of the prepared prostate cDNA. Amplification was carried out in a Perkin Elmer 9600 thermocycler $\left(94{ }^{\circ} \mathrm{C}\right.$ for $9 \mathrm{~min}, 43$ cycles of 30 -s denaturation at $94{ }^{\circ} \mathrm{C}$ and 1 -min annealing/extension at $66{ }^{\circ} \mathrm{C}$; final extension at $66{ }^{\circ} \mathrm{C}$ for 12 min; Perkin Elmer, Norwalk, CT). Then $15 \mu \mathrm{L}$ of this PCR product was visualized on a $2 \%$ agarose gel with ethidium bromide. 


\section{ATGGCCACAGCAGGAAATCCCTGGGGCTGGTTCCTGGGGTACCTCATCCTTGGTGTCG CAGGATCGCTCGTCTCTGGTAGCTGCAGCCAAATCATAAACGGCGAGGACTGCAGCCC GCACTCGCAGCCCTGGCAGGCGGCACTGGTCATGGAAAACGAATTGTTCTGCTCGGGC GTCCTGGTGCATCCGCAGTGGGTGCTGTCAGCCGCACACTGTTTCCAGAACTCCTACAC CATCGGGCTGGGCCTGCACAGTCTTGAGGCCGACCAAGAGCCAGGGAGCCAGATGGT GGAGGCCAGCCTCTCCGTACGGCACCCAGAGTACAACAGACCCTTGCTCGCTAACGAC CTCATGCTCATCAAGTTGGACGAATCCGTGTCCGAGTCTGACACCATCCGGAGCATCAG CATTGCTTCGCAGTGCCCTACCGCGGGGAACTCTTGCCTCGTTTCTGGCTGGGGTCTG CTGGCGAACGGCAGAATGCCTACCGT}

Figure 1 - Sequence of the PCR product amplified by the primers PRSTZ F1 and PRSTZ R1. Underlined bases indicate start codon, and the boldface sequence is exon 1.

\section{Cloning AND SEQUencing OF PCR PRODUCT}

The product was cloned into a PCR 2.1-TOPO vector (Invitrogen, Carlsbad, CA, USA) as prescribed by the manufacturer. Briefly, $4 \mu \mathrm{L}$ of the PCR product was mixed with $1 \mu \mathrm{L}$ of vector, incubated at room temperature for 5 min; $2 \mu \mathrm{L}$ of this reaction was then added to a vial of chemically competent One Shot $E$. coli cells (Invitrogen) followed by $30 \mathrm{~min}$ of incubation. Cells were then heat shocked at $42{ }^{\circ} \mathrm{C}$ for 5 min to induce uptake of the vector, and then chilled on ice for $5 \mathrm{~min}$. Transformed cells were selected for in ampicillin-supplemented SOC medium for $30 \mathrm{~min}$ at $37^{\circ} \mathrm{C} ; 25 \mu \mathrm{L}$ of cells were plated on ampicillin-supplemented LB agar and left to grow overnight at $37^{\circ} \mathrm{C}$. Next, white colonies selected and inoculated into $5 \mathrm{~mL}$ of for ampicillin-LB medium for growth overnight on an orbital shaker at $37^{\circ} \mathrm{C}$. Recombinant plasmids were isolated from these cells by a miniprep procedure (Qiagen, Mississauga, Canada) in which bacterial cells were lysed under alkaline conditions. the lysate is then applied to a silica-based spin column, which adsorbs the plasmid DNA only under high salt concentrations during centrifugation. Following an ethanol wash, adsorbed plasmid was eluted from the column using $10 \mathrm{mM}$ Tris- $\mathrm{HCl}$ at $\mathrm{pH}$ 8.5. Purified plasmid was then sequenced on an automated sequencer (ACGT Corp, Toronto, Canada) using the universal M13 reverse primer.

\section{Results and discussion}

T he sequence of the PCR product is shown in Figure 1. The bold face portion represents exon 1, which contains the start codon (underlined). Exon 1 , as shown here, is identical to the exon 1 predicted by computer analysis. The rest of the sequence corresponds to the known cDNA sequence of prostase/KLK-L1 (2). Based on the knowledge derived from this study, it is now possible to predict the amino acid sequence of the KLK-L1 protein with confidence. Although the biological function of this protein is not yet known, the high mRNA levels found in the prostate and its upregulation in prostate cancer-derived cell culture in response to androgen (2) suggests that it may play a role in the pathogenesis of androgen-dependent prostatic neoplasia. The localization of the actual exon 1 of this gene allows the determination of the whereabouts of its promoter and aids in the finding of important promoter elements including the verification of putative ARE sequence found for KLK-L1 (4). To date, it is not known how well the predicted protein sequence resembles the native protein. However, the confirmation of the presence of exon 1 certainly aids in the design of synthetic polypeptides, which in turn, allows the creation of antiserum that has good potential of recognizing the native protein. Such experimental steps are currently on the way.

\section{References}

1. Yousef GM, Luo L, Diamandis EP. Identification of novel human kallikrein-like genes on chromosome 19q13.3-q13.4. Anticancer Res 1999; 19: 2843-52.

2. Nelson PS, Gan L, Ferguson C, et al. Molecular cloning and characterization of prostase, an androgen-regulated serine protease with prostate-restricted expression. Proc Natl Acad Sci U S A 1999; 96: 3114-19.

3. Yousef GM, Obiezu CV, Luo L, Black MH, Diamandis EP. Prostase/KLK-L1, a new member of the human kallikrein gene family, is expressed in prostate and breast tissues and is hormonally regulated. Cancer Res 1999; 59: 4252-56.

4. Stephenson S-A, Verity K, Ashworth LK, Clements JA. Localization of a new prostate-specific antigen-related serine protease gene, KLK4, is evidence for an expanded human kallikrein gene family cluster on chromosome 19q13.3-13.4. J Biol Chem 1999; 274: 23210-14. 